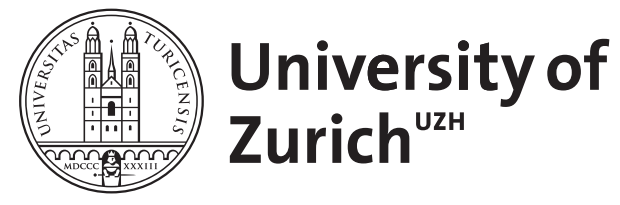
Archive

University of Zurich

University Library

Strickhofstrasse 39

CH-8057 Zurich

www.zora.uzh.ch

Year: 1996

Entwicklung einer Hirntumorbiopsienadel aus faserverstärktem Polymer für die interventionelle MR-Technik

\author{
Reber, $\mathrm{R}$; et al
}

DOI: https://doi.org/10.1515/bmte.1996.41.s1.200

Posted at the Zurich Open Repository and Archive, University of Zurich ZORA URL: https://doi.org/10.5167/uzh-155377

Journal Article

Published Version

Originally published at:

Reber, R; et al (1996). Entwicklung einer Hirntumorbiopsienadel aus faserverstärktem Polymer für die interventionelle MR-Technik. Biomedizinische Technik. Biomedical engineering, 41(s1):200-201.

DOI: https://doi.org/10.1515/bmte.1996.41.s1.200 


\title{
Entwicklung einer Hirntumorbiopsienadel aus faserverstärktem Polymer für die interventionelle MR-Technik
}

\author{
Reber R. ${ }^{1}$, Bernays R.L. ${ }^{2}$, Krayenbühl Ph. ${ }^{1}$, Brandsberg Th. ${ }^{1}$, Brandsberg F. ${ }^{1}$, Romanowski B. ${ }^{3}$, \\ Wintermantel E. ${ }^{1}$, Yonekawa Y. ${ }^{2}$
}

\begin{abstract}
1 Professur für Biokompatible Werkstoffe und Bauweisen, Departement Werkstoffe, ETH Zürich, Schweiz
2 Departement Neurochirurgie, Universitätsspital Zürich, Schweiz

3 Departement Radiologie, MR-Zentrum, Universitätsspital Zürich, Schweiz
\end{abstract}

\section{EINLEITUNG}

Mit einer stereotaktischen Himtumorbiopsie kann minimalinvasiv, sicher und präzis Gewebe einer intrakraniellen Läsion gewonnen werden, um eine histopathologische Diagnose zu stellen. Die konventionelle stereotaktische Biopsie war bisher die Methode der Wahl in diesem Indikationsbereich. Zahlreiche Neuentwicklungen mit dem Ziel der Neuronavigation, intergrieren praeoperativ aufgenommene Bilder immer besser in den eigentlichen Operationsablauf. Der Hauptnachteil dieser indirekten Verfahren besteht darin, dass die praeoperativ aufgenommenen Bilder die anatomischen Verhältmisse während der Operation nicht berücksichtigen. So kommt es z. Bsp. nach der Punktion einer zystischen Läsion zu einer inakzeptablen Verschiebung der anatomischen Verhältnisse. Eine Monitorisierung des eigentlichen Eingriffes ist deshalb nicht möglich.

Das offene interventionelle MR (0.5T, GE Medical Systems, Milwaukee WI) ermöglicht sowohl die Planung wie Monitorisierung eines neurochirurgischen Eingriffs und stellt damit eine bedeutende Vereinfachung mit wesentlich verbesserten Kontrollmöglichkeiten im Vergleich zur konventionellen Stereotaxie dar [1].

Operationen im offenen interventionellen MR erfordern jedoch Instrumente aus MR-kompatiblen Werkstoffen. Dabei müssen mechanische Eigenschaften und Biokompatibilität dieser Werkstoffe die Anforderungen, die an konventionelle Instrumente gestellt werden, ebenfalls erfüllen. Ferromagnetische Werkstoffe sind aufgrund der Kraftwirkung im starken Magnetfeld ausgeschlossen, viele paramagnetsiche Materialien bewirken starke Artefaktbildung (Suszeptibilitätsartefakte).

\section{MATERIAL}

Die in dieser Arbeit entwickelte Hirntumorbiopsienadel besteht aus zwei konzentrisch ineinander laufenden Hohlzylindern. Sie wird im Einsatz primär auf Biegung beansprucht. Das Innenrohr erfährt beim Abscheren des Biopsats zusätzlich eine Torsionsbelastung. Die im gesunden Hirngewebe entstehenden Kräfte sind sehr klein. Erhöhte Biegebeanspruchungen können zum Beispiel dann auftreten, wenn beim Einführen der Nadel ein kalzifizierter Tumor gestreift wird.
Diese Beanspruchungscharakteristik favorisiert den Einsatz eines in axialer Richtung, unidirektional langfaserverstärkten Materials. Deshalb wurde in dieser Arbeit ein mittels Pultrusion hergestellter Werkstoff verwendet. Es handelt sich um hochfeste Kohlenstoffasem (T300) und eine duromere Epoxidmatrix. Der Faservolumengehalt beträgt 65 vol\%. Die kohlenstofffaserverstärkte Biopsienadel ist in Figur la abgebildet.
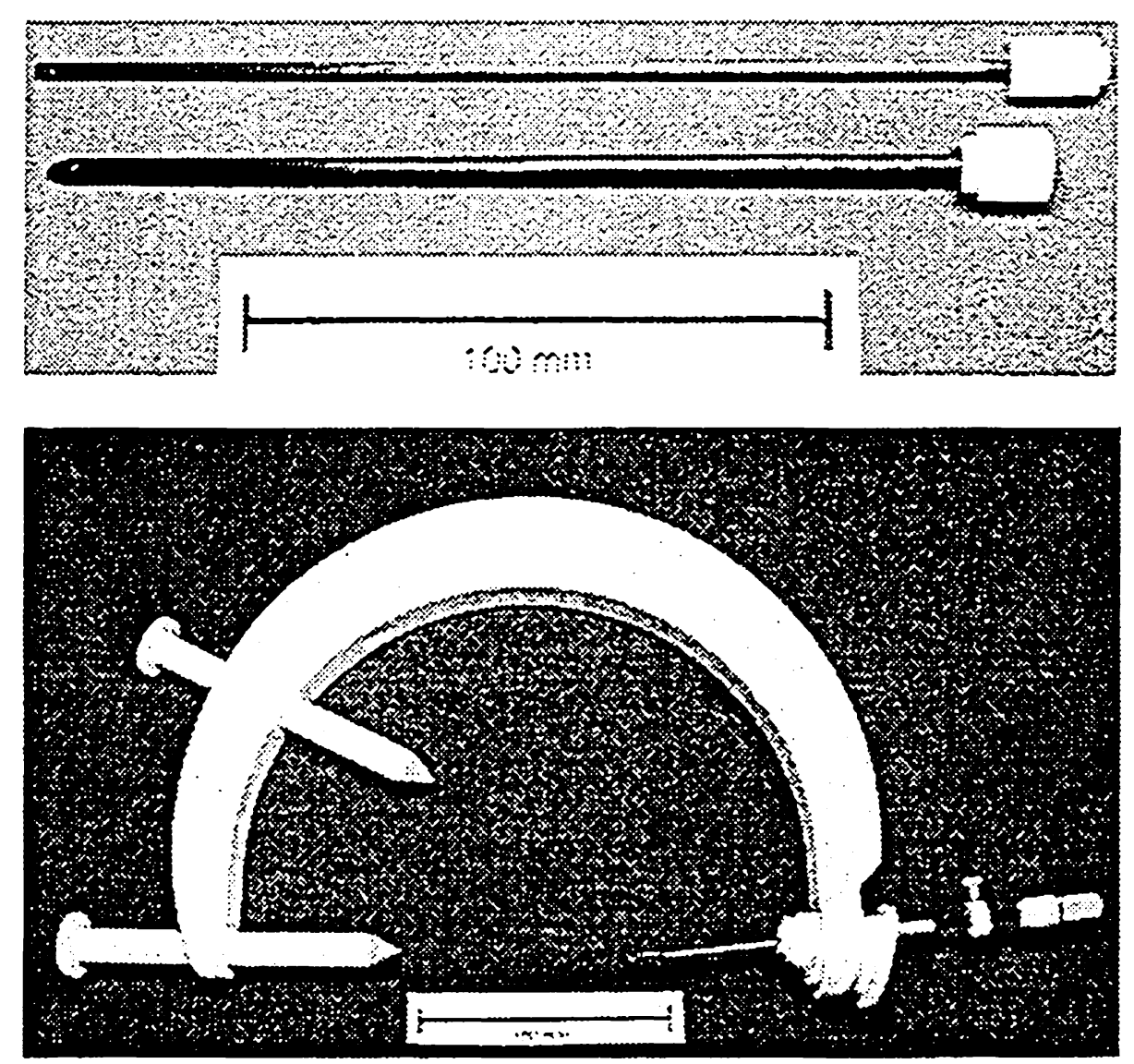

Fig. I $a-b$ :

a: Hirntumorbiopsienadel aus kohlenstoffaserverstärktem Epoxid.

b: Halterungssystem aus PETP mit eingesetzter Nadel

\section{METHODEN}

\section{Herstellung}

Der innere Vollstab wurde beidseitig axial aufgebohrt. Längs des Stabes wurde eine Nute $\left(150 \times 1 \times 1 \mathrm{~mm}^{3}\right)$ gefräst, welche die Applikation eines Vakuums ermöglicht. An der Vorderseite der Innen- und Aussennadel wurden Spitzen aus dem gleichen Werkstoff aufgebracht. Die Dimensionen des äusseren Hohlstabs betragen $4 \mathrm{~mm}$ Aussendurchmesser, $2.5 \mathrm{~mm}$ Innendurchmesser, resp. $0.75 \mathrm{~mm}$ Wandstärke.-Der innere Vollstab weist einen Durchmesser von $2.5 \mathrm{~mm}$ auf. Die 
Gesamtlänge der Nadel beträgt $155 \mathrm{~mm}$. Zusätzlich zur Nadel wurde ein Halterungssystem aus PETP hergestellt (Fig. 1b). Dieses gewährleistet die Fixation und Führung der Nadel während des Eingriffs.

Zur Kontrolle der Nadelposition während der Operation wird ein Aufsatz (Flashpoint 5000, Image Guided Technologies Inc., Bolder, $\mathrm{CO}$ ) benötigt (Fig. 2a). Dieser muss mit hoher Präzision parallel zur Nadelachse fixiert werden.

\section{Charakterisierung}

E-Modul, resp. Bauteilsteifigkeit wurden im Zug- und 4-Pkt-Versuch ermittelt (UMP, Zwick 1456). Mit Hilfe der Finiten Elemente Methode (FEM) wurde die Verteilung der in der Himtumorbiopsienadel auftretenden Torsions- und Biegespannung simuliert. Die Morphologie der Werkstoffoberflächen wurde mittels Rasterelektronen-mikroskopie (REM) untersucht.

\section{Testung}

Die kohlenstoffaserverstärkte, MR-kompatible Hirntumorbiopsienadel wurde in Wassergel, tierischen und menschliche Kadavern auf MR-Kompatibilität (Kontrast, Artefaktbildung) und auf Funktionstüchtigkeit (Dichtheit, Positionierung, Führung) geprüft.

\section{RESULTATE UND DISKUSSION}

\section{Charakterisierung}

Der Biege E-Modul beträgt $110 \mathrm{GPa}$. Dies entspricht einem Zehnfachen des im Pflichtenheft geforderten minimalen Wertes. Kleinere Durchmesser sind daher vom Standpunkt der mechanische Eigenschaften denkbar.

Die FEM Resultate zeigen Spannungsspitzen an den Kerbrändern des inneren Vollstabs. Eine Möglichkeit diese zu umgehen, wäre die Verwendung eines Rohres anstelle eines Vollstabes. Andererseits ist die Herstellung eines faserverstärkten Hohlstabes in diesen Dimension viel komplexer.

Die REM Untersuchungen zeigen die Problematik des hohen Fasergehaltes. Um das Risiko der Faserfreisetzung auszuschliessen wird eine Herabsetzung des Fasergehaltes vorgeschlagen.

\section{Testung}

Die MR-Kompatibilität der verwendeten Werkstoffe ist in Bezug auf Artefaktbildung und Kontrast optimal. Der Einsatz von Kontrastmitteln wäre für die interventionelle Hirntumorbiopsie nicht sinnvoll (Fig. 2b).

Das Übereinstimmen der abgebildeten Position mit der effektiven Position der Nadel wurde über Ventrikelpunktion, Luftinstillation und pathologischer Auswertung des Biopsiekanals überprüft.

Das Gewinnen eines Biopsats erwies sich als problemlos und präzis. Es ist möglich das Aspirieren von Gewebe aufgrund der real-time Bildaquisition direkt zu überprüfen.

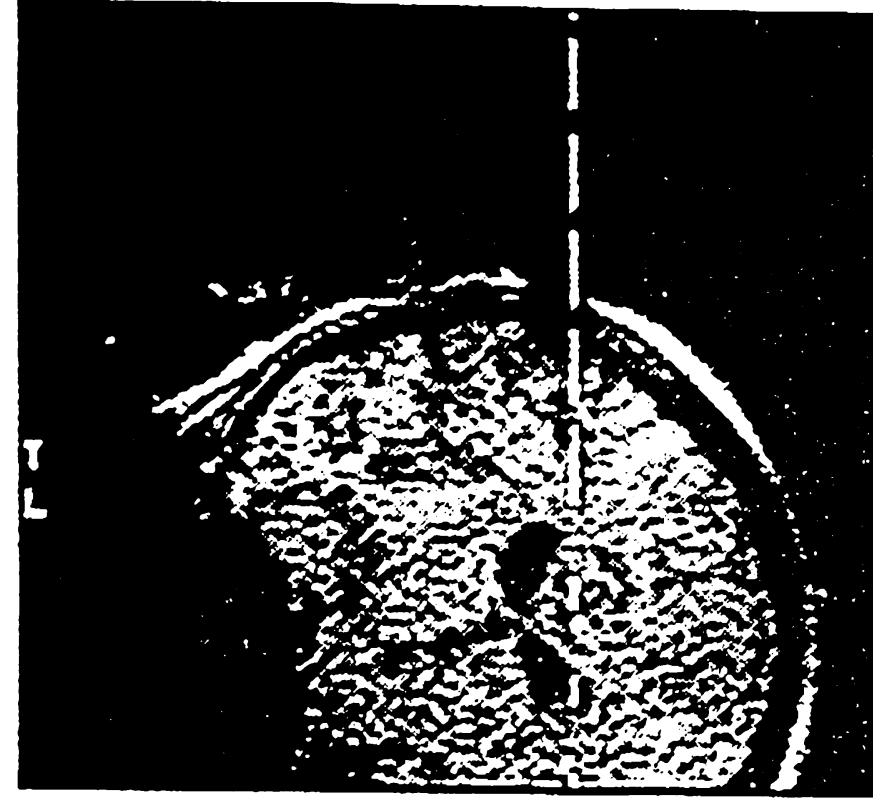

Fig. 2a:

Lokalisierung mittels Flashpoint System, welches die Lage und Spitze des Instruments anzeigt.

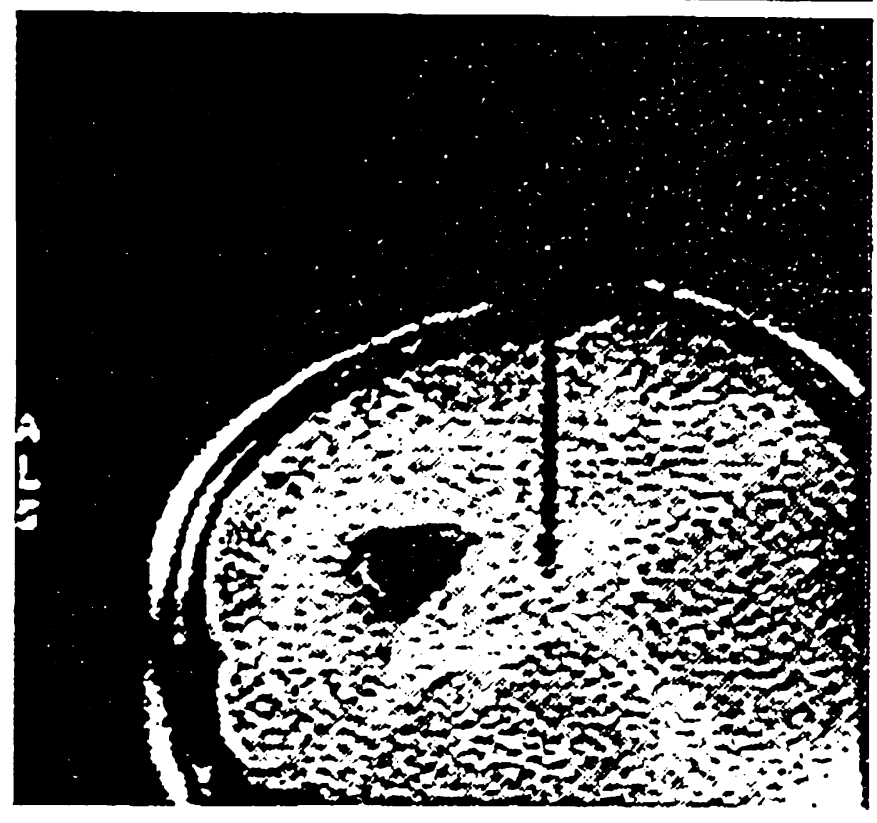

Fig. $2 b$ :

Die kohlenstoffaserverstärkte Hirntumorbiopsie nadel weist einen guten Kontrast zum Hirngewebe ohne Artefaktbildung auf.

\section{SCHLUSSFOLGERUNGEN}

Die im Rahmen dieser Arbeit entwickelte MRkompatible Hirntumorbiopsienadel aus pultrudiertem, kohlefaserverstärktem Epoxid ist für Hintumorbiopsien unter MR-Kontrolle aus folgenden Gründen geeignet:

- Die verwendeten Werkstoffe sind MR-kompatibel. Sie erfahren im Magentfeld keine Kraftwirkung, sind artefaktfrei und werden in realen Dimensionen und Lage abgebildet.

- In Kadaverstudien wurde nachgewiesen, dass eine Biopsie mit hoher Präzision, Sicherheit und unter ständiger Kontrolle durchführbar ist.

Weiterführende Arbeiten sollten sich mit der Optimierung des Werkstoffes und des Bauteils beschäftigen.

Zusammenfassend gilt, dass faserverstärkte Polymere, bedingt durch ihre magnetischen und mechanischen Eigenschaften als Konstruktionswerkstoff für MRkompatibles chirurgisches Instrumentarium geeignet sind.

\section{LITERATUR}

[1] Bernays R.L., Reber R., Wintermantel E., Yonekawa Y., Real-Time Frameless Stereotactic Biopsy in the Open MR: First Developments, EANS Wintermeeting, 2/96, Messina, Italy, Abstracts Book, p. 25. 